\title{
Amyloidogenic variant of apolipoprotein A-I elicits cellular stress by attenuating the protective activity of angiogenin
}

\author{
R Del Giudice ${ }^{1,2}$, DM Monti ${ }^{*, 1,3}$, C Sarcinelli ${ }^{4}$, A Arciello ${ }^{1,3}$, R Piccoli $^{1,3}$ and G-F Hu ${ }^{*, 2}$
}

Amyloidogenic 'gain-of-function' mutations in apolipoprotein A-I (ApoA-I) gene (APOA1) result in systemic amyloidosis characterized by aggregate deposition and eventually cell death. However, how amyloidogenic variants of ApoA-I induce cell death is unknown. Here we report that one of the mechanisms by which amyloidogenic ApoA-I induces cell death is through attenuating anti-stress activity of angiogenin (ANG), a homeostatic protein having both pro-growth and pro-survival functions. Under growth conditions, ANG is located in nucleolus where it promotes ribosomal RNA (rRNA) transcription thereby stimulating cell growth. In adverse conditions, ANG is relocated to cytoplasm to promote damage repairs and cell survival. We find that in cells overexpressing the L75P-APOA1 mutant ANG expression is decreased and normal cellular localization of ANG is altered in response to stress and growth signals. In particular, ANG does not relocate to cytoplasm under stress conditions but is rather retained in the nucleolus where it continues promoting rRNA transcription, thus imposing a ribotoxic effect while simultaneously compromising its pro-survival activity. Consistently, we also find that addition of exogenous ANG protects cells from L75P-ApoA-I-induced apoptosis.

Cell Death and Disease (2014) 5, e1097; doi:10.1038/cddis.2014.45; published online 6 March 2014

Subject Category: Neuroscience

Apolipoprotein A-I (ApoA-I) is a major component of highdensity lipoprotein. ApoA-I has a critical role in lipid metabolism. ${ }^{1}$ It is antiatherogenic and has a protective function against cardiovascular diseases. ${ }^{2}$ ApoA-I is synthesized in the liver and intestine as a pre-pro-protein. After the cleavage of the pre- and pro-peptides, the 28-kDa mature protein is secreted into the plasma, where it can exist in either a lipid-associated or a lipid-free form $(5-10 \%) .{ }^{3}$ Several mutations in apolipoprotein A-I gene (APOA1) have been found. Among them, 19 have been associated with familial systemic amyloidosis, a 'gain-of-function' genetic disease, ${ }^{4}$ which is characterized by deposition of protein aggregates in peripheral organs, such as the heart, liver, kidneys, nerves, ovaries, or testes, leading to organ damage. ${ }^{5}$ Most of the mutations occur as single-nucleotide change, although deletions and/or insertions have also been described. ${ }^{6,7} A P O A 1$ mutations are heterozygous in all ApoA-I-related amyloidosis patients so far investigated so that in these patients both the wild type (WT) and the mutated forms are expressed. However, the plasma level of the mutated forms seems to be lower than that of the WT. ${ }^{4}$ Amyloid fibrils isolated ex vivo are mainly constituted of the N-terminal fragments of 90-100 residue long of the variants with a trace amount of full-length WT-ApoA-I. Fibril formation is a key event in ApoA-I-related amyloidosis, ${ }^{8}$ but the mechanisms by which amyloid fibrils induce cellular stress resulting in cell death is still unclear.

Angiogenin (ANG) has recently emerged as an important stress-regulator that balances cell growth and survival depending on cellular and environmental status. ${ }^{9}$ ANG is a $14-\mathrm{kDa}$ protein belonging to the vertebrate-specific, secreted ribonuclease superfamily. ${ }^{9}$ Under growth conditions, ANG undergoes nuclear translocation and accumulates in nucleolus ${ }^{10}$ where it binds to the promoter region of ribosomal DNA (rDNA) thereby stimulating ribosomal RNA ( $r R N A$ ) transcription. ${ }^{11}$ ANG-stimulated rRNA synthesis is required to meet the high metabolic requirement of actively proliferating cells. ${ }^{12}$ When cells are in adverse conditions, ANG relocates to stress granules in the cytoplasm. ${ }^{13}$ Stress-induced relocalization of ANG from nucleolus to cytoplasm is believed to halt cell growth and promote cell survival. Concurrently, release of nucleolar ANG will decrease rRNA transcription so that growth will slow down to allow adequate time for cells to

\footnotetext{
${ }^{1}$ Department of Chemical Sciences, University of Naples Federico II, via Cinthia 4, Naples 80126, Italy; ${ }^{2}$ Molecular Oncology Research Institute, Tufts Medical Center, 800 Washington Street, Boston, MA 02111, USA; ${ }^{3}$ National Institute of Biostructures and Biosystems (INBB), Rome, Italy and ${ }^{4}$ Department of Biology, University of Naples Federico II, via Cinthia 4, Naples 80126, Italy

*Corresponding author: DM Monti, Department of Chemical Sciences, University of Naples Federico II, via Cinthia 4, Naples 80126, Italy. Tel: +39 081679150; Fax: +39 081679233; E-mail: mdmonti@unina.it

or G-F Hu, Molecular Oncology Research Institute, Tufts Medical Center, 800 Washington Street, Boston, MA 02111, USA. Tel: +1 6176364776; Fax: +1 6176369230; E-mail: ghu@ @uftsmedicalcenter.org

Keywords: Apolipoprotein A-l; amyloidosis; angiogenin; cellular stress; cytoprotection

Abbreviations: ALS, amyotrophic lateral sclerosis; AD, Alzheimer's disease; ANG, angiogenin; APOA1, apolipoprotein A-I gene; ApoA-I, apolipoprotein A-I (protein); $A O$, acridine orange; EB, ethidium bromide; IRES, internal ribosome entry sequence; NLS, nuclear localization sequence; PD, Parkinson's disease; rDNA, ribosomal DNA; rRNA, ribosomal RNA; tiRNA, tRNA-derived, stress-induced small RNA; WT, wild type

Received 24.11.13; revised 03.1.14; accepted 07.1.14; Edited by A Stephanou
} 
adapt to stress conditions. Simultaneously, cytoplasmic ANG mediates the production of a novel class of small RNAs termed tiRNAs, standing for tRNA-derived, stress-induced small RNAs. ${ }^{14}$ tiRNAs suppress cap-mediated global protein translation, ${ }^{14}$ but not internal ribosome entry sequence (IRES)-mediated translation, ${ }^{15}$ which is often used by prosurvival and anti-apoptotic genes. ${ }^{16}$ Thus, relocalization of ANG from nucleus to cytoplasm under stress conditions saves anabolic energy to promote damage repairs and enhance cell survival.

The growth and survival function of ANG has been associated with a number of pathological conditions, including cancers and neurodegenerative diseases. ${ }^{17}$ Loss-of-function mutations in the coding region of $A N G$ gene have been identified in amyotrophic lateral sclerosis (ALS) ${ }^{18,19}$ and Parkinson's disease (PD). ${ }^{20}$ Accumulation of misfolded protein aggregates and the consequent endoplasmic reticulum (ER) stress are a hallmark of neurodegenerative diseases. ANG-mediated stress response is believed to alleviate stress damages inflicted by protein aggregates in neurodegenerative diseases. ${ }^{9}$ Haploinsufficiency of $A N G$ is a risk factor not only for ALS and PD but also for Alzheimer's disease (AD). ANG level is substantially decreased in the serum of ALS patients compared with control subjects. ${ }^{21}$ As fibrillogenesis is a common feature of $A D$ and $P D$, in which a role of ANG has been envisaged, and as fibrillogenesis is an underlying pathogenesis of ApoA-I-related amyloidosis, we hypothesized that pathogenic ApoA-I may suppress ANG expression and that a decrease in ANG level may be a mechanism by which amyloidogenic ApoA-I induce cell death. We transfected HepG2 cells with the cDNA encoding L75PApoA-I, an amyloidogenic mutant that preferentially deposits amyloid fibrils in the liver, and characterized the effect of L75P-ApoA-I on ANG-mediated stress response of the cells. As liver cells per se do express APOA1, this cell model, in which both the WT and the mutated forms are expressed, is a useful experimental system in that it reproduces patient's heterozygosis, thus mimicking a pathological condition. HepG2 cells transfected with vector control and WT-APOA1 were included to mimic healthy condition. Our results show that in cells expressing the amyloidogenic mutant L75PAPOA1 ANG expression and its stress-induced relocalization from nucleolus to cytoplasm are altered. Addition of exogenous ANG can mitigate L75P-ApoA-I-induced apoptotic cell death.

\section{Results}

L75P-ApoA-I variant is accumulated within the cell. Deposition of the L75P-ApoA-I amyloid fibrils occurs preferentially in the liver. ${ }^{8}$ As HepG2 cells express endogenous WT-APOA $1,{ }^{4}$ cells transfected with L75P-APOA1 will simultaneously express both the natural protein and the L75P variant, thus mimicking the heterozygosis nature in human patients. We also transfected HepG2 cells either with WT$A P O A 1$ or with an empty vector and used them as controls mimicking healthy subjects.

Figure 1 shows that ApoA-I proteins were detected in both conditioned media and cell lysates in all three transfectants. In the vector control and WT-APOA1 transfectants, majority of ApoA-I protein was secreted as shown by immunoblot (Figure 1a). Cell-associated ApoA-I was estimated by ImageJ analysis (U.S. National Institutes of Health, Bethesda, MA, USA) to be 8 and $6 \%$, respectively, of the total ApoA-I in vector and WT-APOA1 transfectants (Figure 1b). However, in L75P$A P O A 1$ transfectants, there was a substantially larger portion of ApoA-I protein retained within the cell (Figure 1a). Cell-associated ApoA-I was $20 \%$ of the total protein in L75P-APOA1 transfectants (Figure 1b), representing a $\sim$ three-fold increase in cytosolic retention of ApoA-I. These results demonstrate that expression of the amyloidogenic $A P O A 1$ results in cellular retention of this protein.

L75P-ApoA-I suppresses ANG expression and abolishes its stress-induced upregulation. The effect of the amyloidogenic ApoA-I variant on ANG expression was first examined by quantitative RT-PCR (qRT-PCR). Under growth conditions, ANG mRNA level was unchanged in the cells overexpressing the WT-APOA1 but decreased by $32 \%$ in L75P-APOA1 transfectants (Figure 2a, black bars, $P<0.001)$. It is known that serum withdrawal induces cell stress leading to apoptosis in a variety of cells, including HepG2 cells. ${ }^{22}$ Therefore, we used serum starvation to analyze cellular response to stress conditions and a potential ANG involvement. Cells were cultured in DMEM containing $10 \%$ FBS for $96 \mathrm{~h}$ or for $48 \mathrm{~h}$ in growth conditions and then changed to serum-free medium for another $48 \mathrm{~h}$. We found that upon stress $A N G$ expression was induced in vector control and WT-APOA1 transfectants but not in L75P$A P O A 1$ transfectants. ANG mRNA increased in the vector
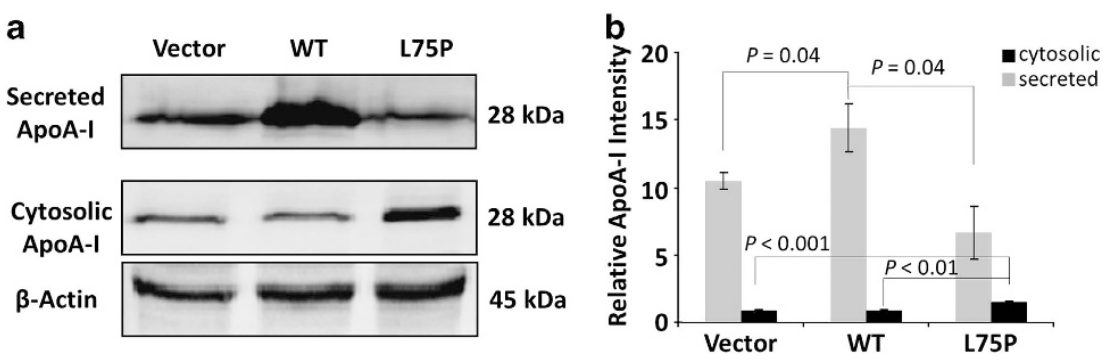

Figure 1 Level of cell-associated and secreted ApoA-I in HepG2 cells. The vector control, WT-APOA1 and L75P-APOA1 transfectants were cultured for 72 h. Conditioned media were collected, cells were detached by trypsinization and counted. (a) Western blotting analysis. Secreted ApoA-I was analyzed from conditioned medium corresponding to 30000 cells. Cell-associated ApoA-I was analyzed from cell lysate of $50 \mu \mathrm{g}$ total proteins (from $\sim 200000$ cells). $\beta$-Actin was blotted as the loading control for cell associated ApoA-I. (b) ImageJ analysis of the band intensity of cytosolic (black) and secreted (grey) ApoA-I. The relative intensity of ApoA-I signal was normalized to $1 \times 10^{6}$ cells. Data shown are the means \pm S.E.M. of three independent experiments 

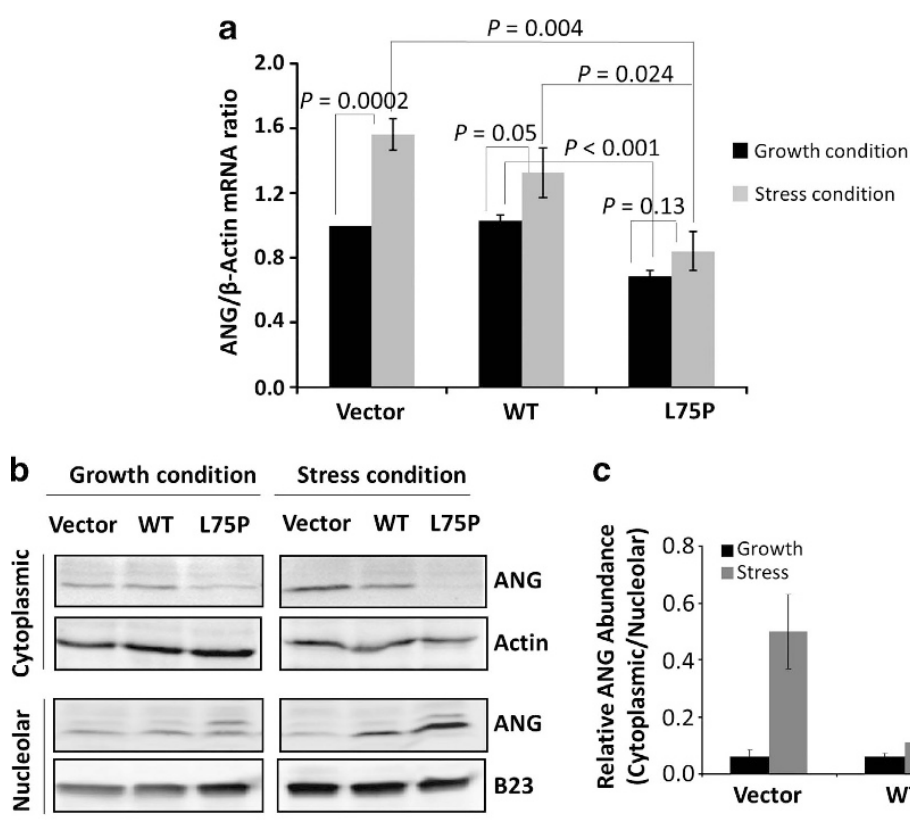

C
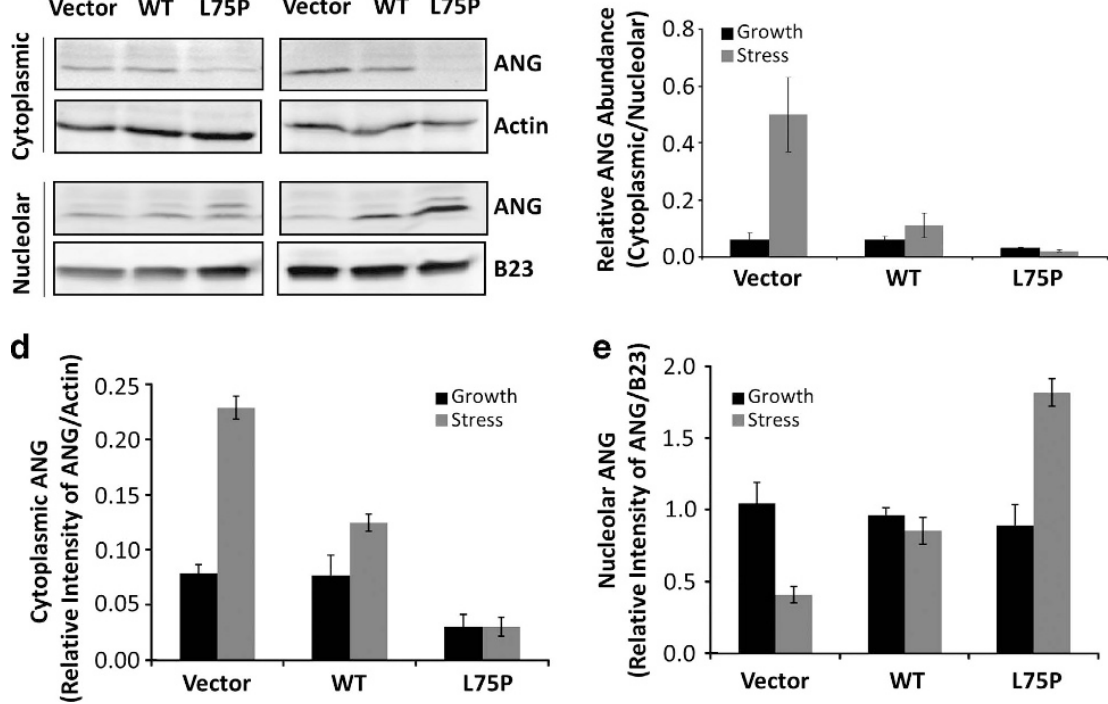

Figure 2 Effect of L75P-ApoA-I on ANG expression and cellular localization. (a) qRT-PCR analysis of ANG mRNA. Cells were cultured in the presence (black bars) or absence (grey bars) of fetal bovine serum (FBS) for $48 \mathrm{~h}$. Total RNA was extracted and analyzed with probes specific for ANG and ACTB mRNAs. $\triangle \triangle C$ Ct method was used. The amount of $A N G$ mRNA was normalized to the endogenous actin and each $\triangle \mathrm{Ct}$ was then normalized to the value of vector control transfectants. Data shown are the means \pm S.D. of three independent experiments. (b) Immunoblot analysis of cytoplasmic and nuclear ANG. Cells were cultured in the presence or absence of FBS for $48 \mathrm{~h}$. Cytoplasmic and nucleolar fractions were obtained by subcellular fractionation. A total of $100 \mu \mathrm{g}$ proteins was loaded in each lane. (c) Ratio of cytoplasmic versus nucleolar ANG. (d) Relative abundance of cytoplasmic ANG normalized to $\beta$-actin. (e) Relative abundance of nucleolar ANG normalized to nucleophosmin B23. Data shown in c-e are the means \pm S.D. of three independent experiments

control and in WT-APOA1 transfectants by $56 \%(P=0.0002)$ and $28 \%(P=0.05)$, respectively (Figure 2a, grey bars). However, serum withdrawal induced only an insignificant increase in ANG expression in L75P-APOA1 transfectants $(15 \%, P=0.13)$. These results indicate that the amyloidogenic ApoA-I variant suppresses ANG expression both in growth and adverse conditions. Furthermore, it abolishes stress-induced upregulation of $A N G$.

We also found that tunicamycin, an ER stress inducer, fails to induce a significant upregulation of ANG in L75P-APOA1 transfectants (Supplementary Figure S1), indicating that the suppressive effect of amyloidogenic ApoA-I variant on ANG expression is not limited to a certain type of stress.

ANG is retained in the nucleoli in L75P-APOA1 transfectants. Figure $2 \mathrm{~b}$ shows the immunoblot analysis of ANG in the cytoplasmic and nucleolar fractions of three HepG2 transfectants. Under normal growth conditions, ANG was detectable in both cytoplasmic and the nucleolar fractions in all three transfectants (lanes 1-3). The ratio of cytoplasmic versus nucleolar ANG was 0.06 in the vector control and in WT-APOA1 transfectants and 0.03 in L75P$A P O A 1$ transfectants (Figure 2c, black bars). Thus, not only ANG expression is decreased in L75P-APOA 1 transfectants but also the ratio of cytoplasmic to nucleolar ANG decreased by two-fold. When cells were cultured in serum-free conditions, there was a significant increase of cytoplasmic ANG (Figure 2d) with a concomitant decrease of nucleolar ANG (Figure 2e) in the vector control and in WT-APOA1 transfectants, which resulted in a significant increase in the ratio of cytoplasmic to nucleolar ANG (Figure 2c, grey bars). However, no increase in cytoplasmic ANG was observed in L75P-APOA1 transfectants (Figure 2d). Instead, majority of cellular ANG was detected in the nucleolar fraction, resulting in a two-fold increase in nucleolar ANG (Figure 2e). As a consequence, the ratio of cytoplasmic to nucleolar $A N G$ decreased rather than increased in L75P-APOA1 transfectants under stress conditions (Figure 2c).

Similar observations were obtained under different types of stresses, including ER stress induced by 
bredfeldin A (Supplementary Figure S2) and tunicamycin (Supplementary Figure S3) and oxidative stress induced by sodium arsentite (Supplementary Figure S3). These results indicate that expression of L75P-APOA1 mutant results in nucleolar retention of ANG under adverse growth conditions. As stress-induced relocalization of ANG from nucleolus to cytoplasm is important for its cytoprotective activity, ${ }^{13}$ alteration of this process could be one of the reasons by which amyloidogenic ApoA-I variant elicits cell death.

Alterations of ANG subcellular localization in cells expressing amyloidogenic ApoA-I were confirmed by indirect immunofluorescence. Confocal microscopic images showed that ANG was detected mainly in the nucleoli in the vector control HepG2 transfectants under normal growth conditions (Figure 3a, arrows). When cells were cultured in serum-free medium, ANG was detected mainly in the cytoplasm (Figure 3b, dashed arrows). Nucleolar and cytoplasmic localization of ANG under growth and stress conditions, respectively, was conserved in the cells overexpressing WTAPOA1 (Figures $3 \mathrm{c}$ and $\mathrm{d}$ ). It is notable that ANG was still detectable in the nucleoli under stress conditions (Figure $3 \mathrm{~d}$, arrows), suggesting that WT-APOA1, when overexpressed, is also pathogenic, even though not as detrimental as the amyloidogenic mutant. In cells that express L75P-APOA1, only a faint staining of nucleolar ANG was observed, whereas strong cytoplasmic staining was observed (Figure 3e, dashed arrows). Most significantly, nucleolar staining of ANG did not decrease but was rather enhanced when cells were cultured in serum-free medium (Figure $3 f$, arrows). At the same time, a concomitant decrease in cytoplasmic ANG was observed. Thus, there is an opposite traffic of ANG between the cytoplasmic and nucleolar compartments in cells expressing the amyloidogenic L75P-APOA 1 mutant in response to stress.

Amyloidogenic ApoA-I increases cell susceptibility to stress. ANG is known to have an important role in both cell growth $^{17}$ and survival. ${ }^{9}$ The growth and survival function of ANG is regulated by its differential cellular localization. ${ }^{13}$ The findings that L75P-ApoA-I inhibits stress-induced relocalization suggest that amyloidogenic ApoA-I may affect stress response of the cells. We first examined the effect of serum starvation on cell numbers. Figure 4 shows that there is no difference in cell numbers among the vector (Figure $4 \mathrm{a}$ ), WT-APOA1 (Figure 4b), and L75P-APOA1 (Figure 4c) transfectants when they were cultured under normal growth conditions (Figure 4, black lines). When cells were cultured in serum-free medium, cell numbers were significantly decreased in all three transfectants, but the percentage of decrease was much more dramatic in L75P-APOA1 transfectants (Figure 4, blue lines). For example, at the 72-h time point, cell numbers of the vector control, WT-APOA1, and L75P-APOA1 transfectants under serum-free condition was 58,53 , and $25 \%$, respectively, of that under growth condition (Figure 4).

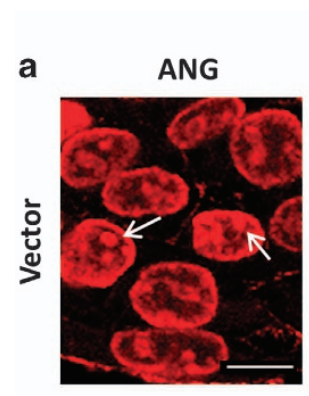

\section{Growth condition}
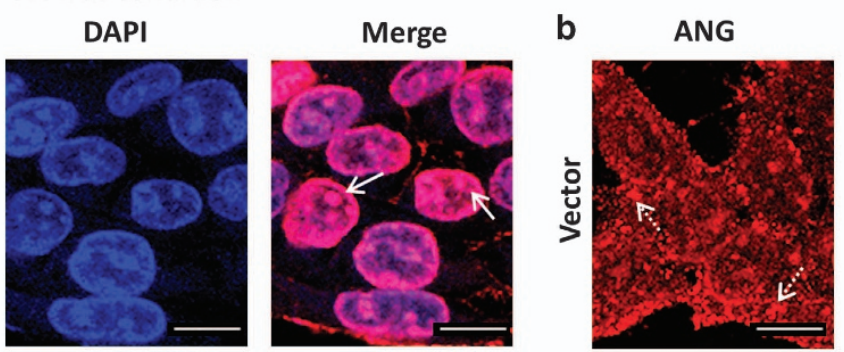

\section{Stress condition}
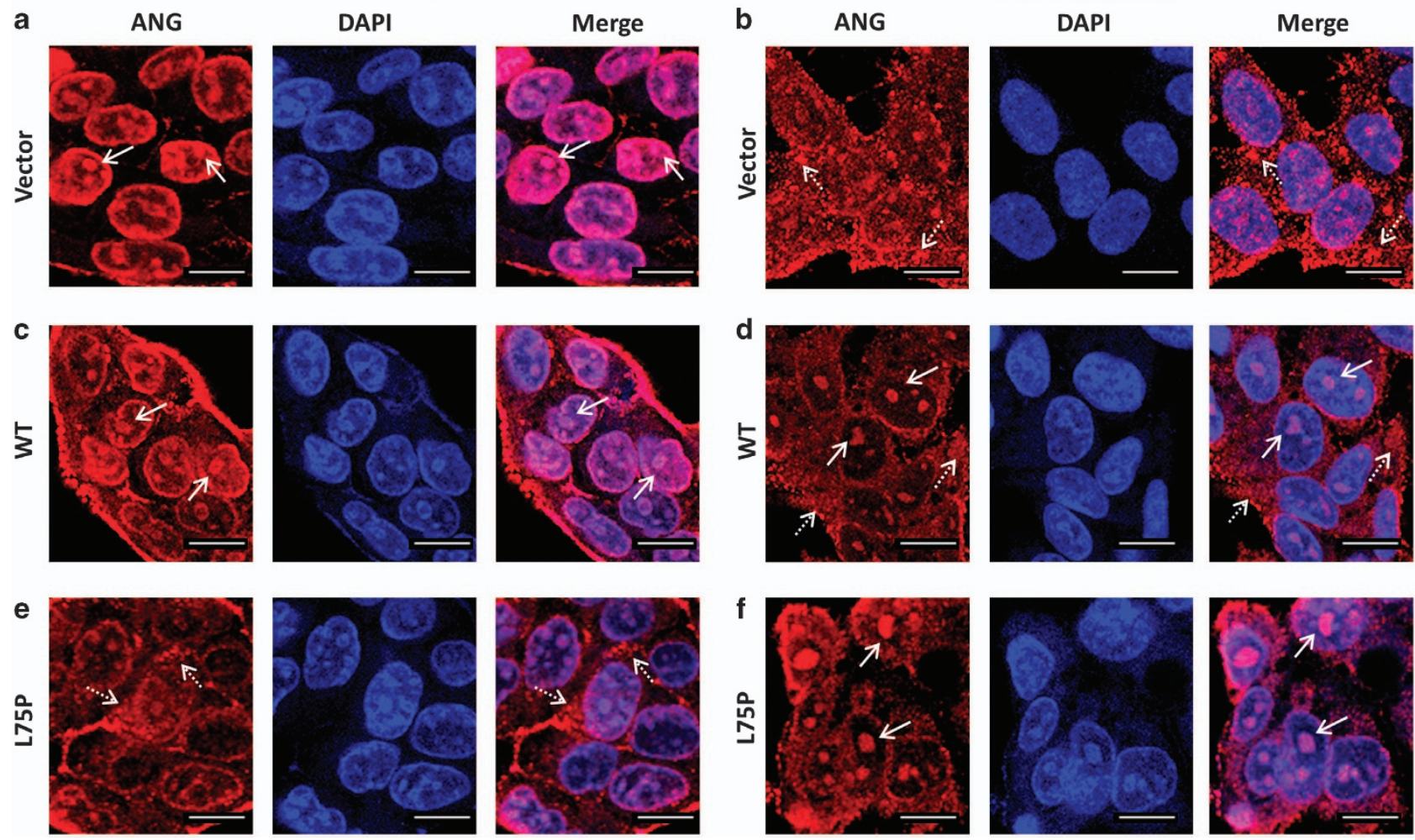

Figure 3 Confocal microscopy analyses of subcellular localization of ANG. (a and b) Vector control, (c and d) WT-APOA1, and (e and f) L75P-APOA1 transfectants of HepG2 cells were cultured in normal growth medium $(\mathbf{a}, \mathbf{c}, \mathbf{e})$ or in serum-free medium $(\mathbf{b}, \mathbf{d}, \mathbf{f})$, fixed, and stained by ANG mAb 26-2F and Alexa 610-labeled goat anti-mouse $\mathrm{F}(\mathrm{ab})_{2}$. Nuclei were stained with Hoechst 33342 . Confocal images were taken with a Leica TCS SP5 confocal microscope. The images shown are the middle panels of the $Z$-sections. Nucleolar and cytoplasmic ANG are indicated by arrows and dashed arrows, respectively. Scale bar: $20 \mu \mathrm{m}$. DAPI, 4,6-diamidino-2-phenylindole 

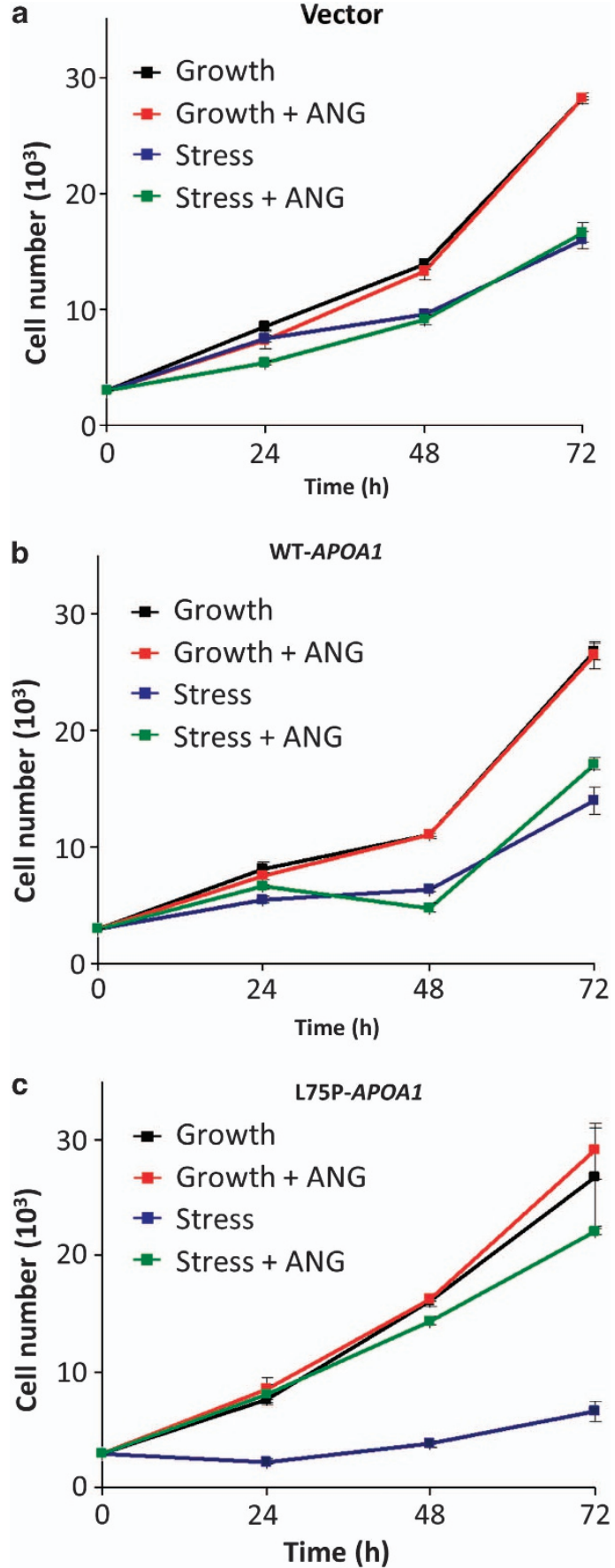

Figure 4 Effect of L75P-ApoA-I on cell growth. (a) The vector control, (b) WTAPOA1, and (c) L75P-APOA1 transfectants were cultured in normal growth medium (Dulbecco's modified Eagle's medium (DMEM) $+10 \%$ fetal bovine serum) (black and red lines) or in serum-free DMEM (green and blue lines) in the absence (black and blue lines) or presence (red and green lines) of $0.5 \mu \mathrm{g} / \mathrm{ml} \mathrm{ANG} \mathrm{for} \mathrm{different}$ lengths of time $(24,48,72 \mathrm{~h})$. Cell numbers were determined by MTT (3-[4,5dimethylthiazol-2-yl]-2,5 diphenyl tetrazolium bromide) assay. Data shown are the means \pm S.E.M. of three independent experiments

In agreement with a reduced cell growth, we found that in cells expressing the amyloidogenic mutant apoptosis was significantly increased, under both growth and stress conditions (Figure 5). Ethidium bromide (EB) and acridine orange
(AO) staining was used to identify apoptotic cells. ${ }^{23}$ AO permeates intact cells and will stain all the nuclei in green, whereas EB enters cells only when the plasma membrane is damaged and therefore stain apoptotic cells in red. Figure 5 shows that cell apoptosis was significantly increased in L75P-APOA1 transfectants under both growth and stress conditions. The portion of EB-positive cells was $20.7 \pm 3.2$ (Figure 5a), $19.9 \pm 3.5$ (Figure 5e), and $35.8 \pm 3.1 \%$ (Figure 5i), respectively, in vector control, WT-APOA1, and L75P-APOA 1 transfectants under normal culture conditions and was $48.7 \pm 3.4$ (Figure $5 \mathrm{c}$ ), $49.0 \pm 3.0$ (Figure $5 \mathrm{~g}$ ), and $62.5 \pm 2.4 \%$ (Figure $5 \mathrm{k}$ ) under stress conditions. These results suggest that lower cell growth of L75P-APOA1 transfectants is the result of increased apoptosis under stress conditions. It is unclear at present why the increased apoptosis of cells expressing the amyloidogenic mutant grown in normal conditions was not correlated to a reduced cell growth.

ANG rescues stress-induced apoptosis in L75P-APOA1expressing cells. Addition of exogenous ANG had no effect on cell proliferation in vector control and WT-APOA1 transfectants, either under growth or stress conditions (Figures $4 \mathrm{a}$ and $\mathrm{b}$ ). This observation is consistent with previous reports that in cancer cells the endogenous ANG level is adequate to meet the high metabolic demand. ${ }^{24,25}$ However, exogenous ANG significantly increased cell number of L75P-APOA1 transfectants cultured under serum-free conditions. The number of cells of L75P-APOA1 transfectants in the presence of ANG was four-fold higher (Figure 4c, green line) than that in the absence of ANG (Figure 4c, blue line) and was similar to that of vector (Figure $4 a$, green line) and WT-APOA1 (Figure 4b, green line) transfectants under the same conditions. Thus, the inhibitory effect of L75PApoA-I on cell growth was completely rescued by exogenous ANG. These results are consistent with the observation of exogenous ANG on cell apoptosis of the three transfectants under growth and stress conditions (Figure 5). Under growth condition, ANG had no effect on vector control and WTAPOA1 transfectants but caused a marginal $(P=0.05)$ reduction in apoptosis from $35.8 \pm 3.1$ (Figure $5 \mathrm{i}$ ) to $29.8 \pm 1.5 \%$ (Figure $5 \mathrm{j}$ ) in L75P-APAO1 transfectants. When cells were cultured in serum-free medium, exogenous ANG reduced apoptosis in L75P-APOA1 transfectants by $30 \%$ $(P<0.0001)$ from $62.5 \pm 2.4$ (Figure $5 \mathrm{k}$ ) to $44.4 \pm 1.2 \%$ (Figure $5 \mathrm{I}$ ). In contrast, exogenous ANG only slightly reduced cell apoptosis in vector control from $48.7 \pm 3.4$ (Figure $5 \mathrm{c}$ ) to $41.6 \pm 2.4 \%$ (Figure $5 \mathrm{~d})(P=0.056)$ and in WT-APOA1 from $49.0 \pm 3.0$ (Figure $5 \mathrm{~g})$ to $44.0 \pm 3.1 \%$ (Figure $5 \mathrm{~h})(P=0.136)$ transfectants. Taken together, these results indicate that exogenous ANG normalizes apoptosis and effectively alleviates the detrimental effect of the amyloidogenic ApoA-I variant on cell survival.

Nuclear translocation and ribonucleolytic activity of ANG is not required for its cytoprotective activity against amyloidogenic ApoA-I. ANG has a classic nuclear translocation sequence (nuclear localization sequence (NLS)), consisting of R31, R32, and R33, which mediates nuclear import. ${ }^{10}$ Mutations at the NLS of ANG inhibit its 

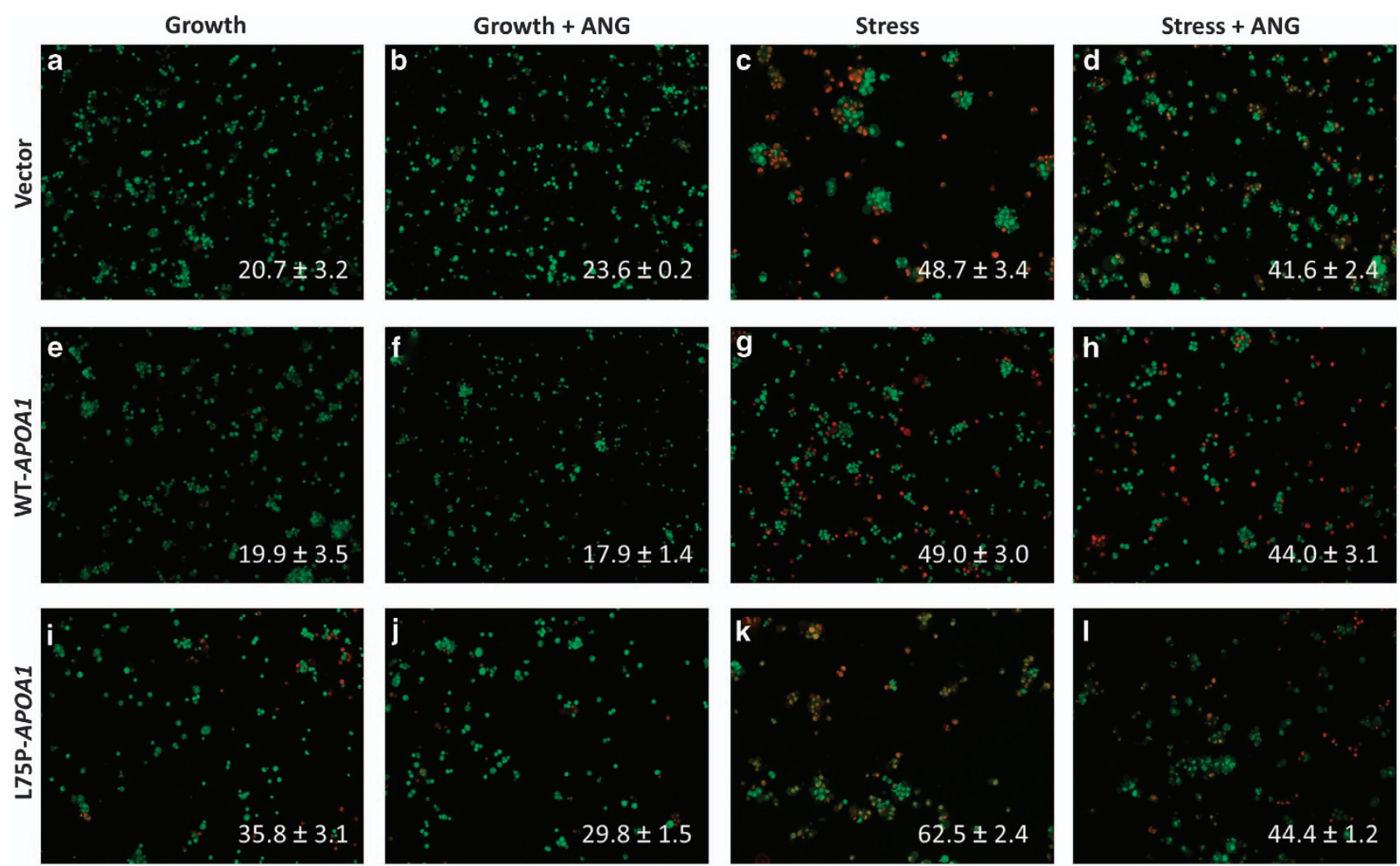

Figure 5 Effect of L75P-ApoA-I on cell apoptosis. (a-d) The vector control, (e-h) WT-APOA1, and (i-I) L75P-APOA1 transfectants were cultured in normal growth medium $(\mathbf{a}, \mathbf{b}, \mathbf{e}, \mathbf{f}, \mathbf{i}, \mathbf{j})$ or in serum-free medium $(\mathbf{c}, \mathbf{d}, \mathbf{g}, \mathbf{h}, \mathbf{k}, \mathbf{l})$ in the presence $(\mathbf{b}, \mathbf{d}, \mathbf{f}, \mathbf{h}, \mathbf{j}, \mathbf{l})$ or absence $(\mathbf{a}, \mathbf{c}, \mathbf{e}, \mathbf{g}, \mathbf{i}, \mathbf{k})$ of $0.5 \mu \mathrm{g} / \mathrm{ml}$ ANG for $48 \mathrm{~h}$. Cells were collected and stained with EB (red) and AO (green). EB-stained cells were counted in a total of 750 cells from five randomly selected areas of each slide. The percentage of apoptotic cells is shown at the bottom of each image. Data shown are the means \pm S.E.M. of triplicates of a representative experiment of at least three repeats

nuclear translocation but do not affect cell surface binding and nucleolytic activity. ${ }^{26} \mathrm{ANG}$ is also an active ribonuclease with a typical catalytic center composed of $\mathrm{H} 13, \mathrm{~K} 40$, and H114. ${ }^{27}$ Inactivation of the ribonucleolytic activity by mutagenesis or chemical modification abolishes angiogenic activity of ANG but has no effect on receptor binding. ${ }^{28}$ In order to know whether nuclear translocation or the ribonucleolytic activity of ANG is required for its protective activity against amyloidogenic ApoA-I, we tested the effects of R33A and K40Q ANG variants on cell proliferation under growth and stress conditions. R33A is not able to translocate to the nucleus, and $\mathrm{K} 40 \mathrm{Q}$ is enzymatically inactive. As expected, both R33A and K40Q ANG variants had no effect on cell proliferation of vector and WT-APOA1 transfectants under both growth and stress conditions (Figures 6a-d). R33A and K40Q had a marginal effect on L75P-APOA1 transfectants under growth conditions but markedly enhanced cell proliferation under stress conditions (Figures $6 e$ and f). At the 72-h time point, proliferation of L75P-APOA1 transfectants was increased by R33A and K40Q by $368(P=0.0004)$ and $382 \%(P=0.0006)$, respectively. These results clearly indicate that nuclear translocation and the ribonucleolytic activity of ANG are not required for the protective activity against amyloidogenic ApoA-I.

rRNA transcription is deregulated in cells expressing amyloidogenic APOA1. An important function of nucleolar
ANG is to bind to the promoter region of rDNA ${ }^{29,30}$ thereby promoting rRNA transcription. ${ }^{11}$ Physical presence of ANG in the nucleolus controls overall activity of rRNA transcription. As ANG was found to be retained in the nucleolus in L75P-APOA1 transfectants under stress conditions (Figures 2 and 3 ), we examined rRNA transcription levels by qRT-PCR in vector control, WT, and L75P-APOA1 transfectants under both growth and stress conditions. Under growth conditions, rRNA transcription in WT and L75P-APOA1 transfectants was $84(P=0.22)$ and $53 \%(P=0.02)$, respectively, of that in vector control transfectants (Figure 7 , black bars). Thus, overexpression of WT-APOA1 did affect rRNA transcription but L75P-ApoA-I significantly decreased rRNA transcription. These results are in agreement with the findings that nucleolar ANG was substantially decreased in L75P-APOA1 but not in WT-APOA1 transfectants (Figure 3). Under serumfree conditions, rRNA transcription was substantially decreased $(44 \%, P=0.03)$ in vector control transfectants, in agreement with relocalization of ANG from nucleolus to cytoplasm. However, in cells that overexpress WT-APOA1, rRNA transcription was only slightly decreased in stress conditions. This is consistent with the fact that a substantial amount of ANG still remains in the nucleoli of these cells under these conditions as shown in Figures 2 and 3 . These results also point out that overexpression of WT-APOA1 can be still pathological, as rRNA transcription is deregulated in response to stress. More significant deregulation of rRNA 

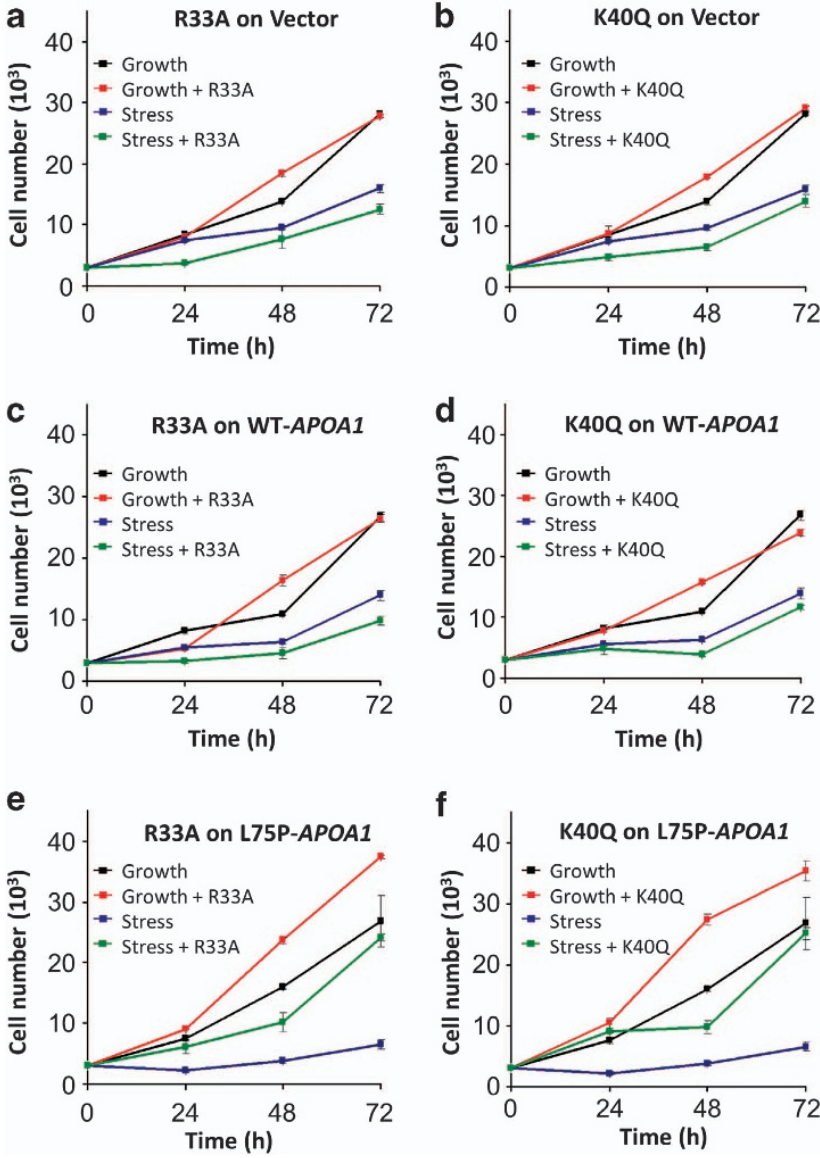

Figure 6 Effect of R33A and K40Q ANG variants on cell proliferation. (a and $\mathbf{b}$ ) The vector control, (c and d) WT-APOA1, and (e and f) L75P-APOA1 transfectants were cultured in complete medium (black and red lines) or in serum-free medium (blue and green lines) in the presence (red and green lines) or absence (black and blue lines) of $0.5 \mu \mathrm{g} / \mathrm{ml} \mathrm{R} 33 \mathrm{~A}(\mathbf{a}, \mathbf{c}, \mathbf{e})$ or $\mathrm{K} 40 \mathrm{Q}(\mathbf{b}, \mathbf{d}, \mathbf{f})$ for 24,48 , and $72 \mathrm{~h}$. Cell numbers were determined by MTT (3-[4,5-dimethylthiazol-2-yl]-2,5 diphenyl tetrazolium bromide) assay. Data shown are means \pm S.E.M. of three independent experiments

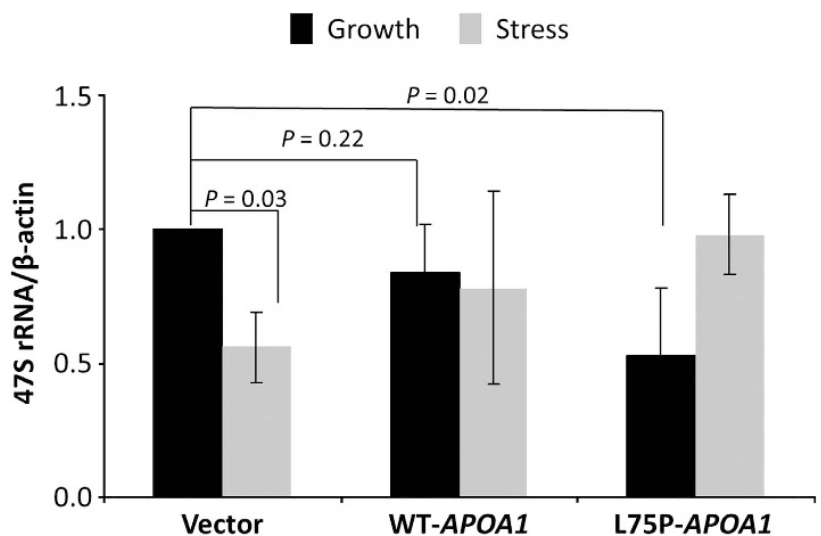

Figure 7 qRT-PCR analysis of $47 \mathrm{~S}$ rRNA level. Cells were cultured in the presence (black bars) or in the absence (grey bars) of serum for $48 \mathrm{~h}$. Total RNA was extracted, reverse transcribed, and analyzed with probes specific for $47 \mathrm{~S}$ rRNA. $\beta$-Actin mRNA was used as internal control. Data shown are the means \pm S.D. of three independent experiments was observed in L75P-APOA1 transfectants. The level of 47S rRNA was increased rather than decreased when cells were cultured in serum-free conditions (Figure 7). This observation is consistent with the finding that nucleolar ANG was increased rather than decreased in these cells. As rRNA transcription is an energy costly process, enhanced rRNA transcription under stress conditions is likely to impose a ribotoxic stress exacerbating cell death. ${ }^{31}$

\section{Discussion}

Accompanied with cytosolic accumulation and decreased secretion of ApoA-I protein (Figure 1), L75P-APOA1 transfectants had higher apoptosis compared with vector and WTAPOA1 transfectants, especially under stress conditions (Figure 5). Downregulation of ApoA-I secretion has previously been observed in transiently transfected cells overexpressing L75P- and L174S-APOA 1. ${ }^{32}$ It is conceivable that the expression of APAO1 mutants is per se a stress factor as the variants will accumulate inside the cell. Abnormal accumulation of cytosolic proteins will not only impede the ability of cells to survive adverse conditions but will also elicit a direct ER stress to the cells. At present, it is unknown whether cytoplasmic accumulation of the mutant forms of ApoA-I is the reason for their cytotoxicity. However, our results have clearly demonstrated that alteration of ANG expression and subcellular localization is one of the reasons.

Subcellular localization of ANG is normally well regulated. Under growth conditions, ANG is mainly located in the nucleolus where it binds to the promoter region of rDNA and stimulates rRNA transcription. ${ }^{29,30}$ Nucleolar localization of ANG and the consequent promotion of rRNA synthesis is a metabolic requirement for actively growing cells, ${ }^{17}$ as a constant supply of rRNA is essential for ribosome biogenesis $^{33}$, which is the key for protein translation and cell growth. ${ }^{34}$ When cells are in adverse conditions, such as nutrient deficiency, oxidative, or ER stress, ANG is no longer located in nucleolus but rather in cytoplasm. ${ }^{13}$ Relocation of ANG from nucleolus to cytoplasm promotes cell survival. On the one hand, it ensures no unnecessary rRNA synthesis so that the precious energy resources are not wasted. On the other hand, cytoplasmic ANG mediates the production of tiRNAs, ${ }^{14}$ which have been shown to suppress global protein translation so that anabolic energy is further saved. ${ }^{15}$ tiRNAs have no effect on IRES-mediated protein translation, a mechanism often used by pro-survival and anti-apoptotic mRNAs such as $B C L-2, X I A P$, and $H I F-1 \alpha .{ }^{35}$ Therefore, relocation of ANG from the nucleolus to the cytoplasm in response to stress is an important survival mechanism of the cells under adverse conditions. We found that this stress response is lost in the cells expressing an amyloidogenic APOA1 mutant. In HepG2 cells overexpressing the L75PAPOA1 mutant, more nucleolar ANG was observed under stress conditions than under normal growth conditions (Figures 2 and 3). As a consequence of abnormal accumulation of nucleolar ANG, rRNA is continuously transcribed when cells are under stress (Figure 7), which imposes a ribotoxic effect to the cells ${ }^{31}$ and eventually results in cell apoptosis. These results indicate that suppression of ANG expression and alteration of its subcellular localization is a hallmark 
pathogenesis of amyloidogenic ApoA-I. We therefore reasoned that, if this hypothesis is correct, addition of exogenous ANG to serum-free medium should be able to restore cell viability. We found a strong anti-apoptotic effect of ANG on L75P-APOA1-expressing HepG2 cells (Figures 4 and 5). This is in line with previous findings that exogenously added ANG is located in the cytoplasm of stressed cells and that cytoplasmic ANG has a protective role against stress. ${ }^{13}$ Consistently, R33A ANG variant, which is unable to undergo nuclear translocation and therefore accumulates in the perinuclear region, ${ }^{26}$ has full protective activity as does WT ANG (Figure 6). An interesting finding emerges from the analysis of K40Q ANG, an enzymatically inactive variant due to the substitution of GIn for Lys at the enzymatically active site. This variant also retains a full protective activity (Figure 6), indicating that ribonucleolytic activity is not essential for the protective activity of ANG against L75P-ApoA-I-induced cell death. This result also suggests that tiRNAs are not the mediator for enhanced cell survival in this case, as a full ribonucleolytic activity of ANG is required for the production of tiRNAs and for the subsequent reprogramming of protein translation. ${ }^{14,36}$ One possible mechanism for the cytoprotective activity of ANG against amyloidogenic ApoA-I could be through the AKT pathway. ANG is known to activate AKT by binding to cell surface, ${ }^{37,38}$ and it has been reported that ANG protects motor neuron degeneration in $S O D 1^{G 93 A}$ mice through activation of AKT. ${ }^{39}$

It is unclear at present how $A N G$ expression is reduced in L75P-APOA1 transfectants. Suppression of ANG expression by fibrillogenic proteins has been previously reported. For example, Ang1 has been found to be the most downregulated gene in a mouse PD model that overexpresses $\alpha$-synuclein. ${ }^{40}$ It has been shown that $\alpha$-synuclein inhibits histone acetylation, thereby suppressing protein translation, ${ }^{41}$ therefore $\alpha$-synuclein overexpression could cause a reduction in ANG levels via this mechanism. A similar mechanism has been found in another neurodegenerative disease involving the fibrillogenic protein ataxin-3. ${ }^{42}$ It is still unknown whether the amyloidogenic ApoA-I variant suppresses ANG expression through affecting histone acetylation. In any event, downregulation of $A N G$ seems to be a common feature shared by a number of diseases where fibrillogenic proteins are responsible for pathogenesis as has been shown in $A L S,{ }^{43} A D,{ }^{21}$ $\mathrm{PD},{ }^{40}$ and now in ApoA-I associated amyloidosis.

\footnotetext{
Materials and Methods

ANG and variants. WT $A N G$ and the R33R and $K 40 Q$ variants were prepared as recombinant proteins in an Escherichia coli expression system and were purified to homogeneity by the methods described previously. ${ }^{44-46}$

Cell cultures and transfection. HepG2 cells were seeded on six-well plates at a density of $2 \times 10^{5}$ cells/well and cultured in DMEM $\pm 10 \%$ FBS for $24 \mathrm{~h}$. Cells were then washed with Opti-MEM and transfected with empty plasmid pRC-rsv or that encodes either WT-APOA1 or L75P-APOA1 in the presence of Lipofectamine. Stable transfectants were selected by $0.8 \mathrm{mg} / \mathrm{ml} \mathrm{G} 418$.
}

Immunoblot of ApoA-I. Transfectants were cultured on six-well plates at $1 \times 10^{5}$ cells/well in DMEM $\pm 10 \%$ FBS for $72 \mathrm{~h}$. Conditioned media were collected, and cells were detached by trypsinization. Cell numbers were determined with a hemocytometer using trypan blue exclusion assay. Aliquots corresponding to $50 \mu \mathrm{g}$ cytosolic proteins (cell lysate) or to 30000 cells (conditioned medium) were separated on a $15 \%$ SDS-PAGE, and western blotting analysis was carried out with an ApoA-I antibody (Dako, Glostrup, Denmark; 1:500 dilution) and actin antibody (Sigma-Aldrich, St. Louis, MO, USA; 1:1000 dilution) as a loading control for the cytosolic proteins.

Preparation of nucleolar fractions. Nucleolar fractions were prepared according to the method described by Busch. ${ }^{47}$ Nucleolar proteins were extracted by lysing the nucleolar fraction in RIPA buffer (50 mM Tris, pH 8.0, $150 \mathrm{mM} \mathrm{NaCl}, 1 \%$ NP40, $0.5 \%$ deoxycholate, $0.1 \%$ SDS, and $1 \times$ protease inhibitor cocktail). Upon $30 \mathrm{~min}$ incubation on ice, the lysates were centrifuged at $1500 \times \mathrm{g}$ for $10 \mathrm{~min}$ at $4{ }^{\circ} \mathrm{C}$. Following the determination of protein content by the BCA assay, $50 \mu \mathrm{g}$ of proteins were separated on a 4-20\% SDS-PAGE and analyzed by immunoblot with ANG antibody R113 $(1 \mu \mathrm{g} / \mathrm{ml})$ or B23 antibody $(0.3 \mu \mathrm{g} / \mathrm{ml})$ as a loading control.

MTT assay. Cells were seeded in 96-well plates (100 $\mu /$ well) at a density of $3 \times 10^{3} /$ well. After $24 \mathrm{~h}$, cells were cultured for different lengths of time either in complete medium (DMEM $+10 \% \mathrm{FBS}$ ) or in serum-free DMEM in the absence or presence of $0.5 \mu \mathrm{g} / \mathrm{ml}$ ANG (WT, R33A, or K40Q). Cell viability was assessed by MTT assay. For each cell line, a calibration curve was obtained by plating an increasing number of cells per well $\left(0.5-7 \times 10^{3}\right)$.

Immunofluorescence and confocal microscopy. Cells were cultured on cover slips placed inside the culture dishes in the presence or absence of FBS for $72 \mathrm{~h}$, fixed in methanol at $-20^{\circ} \mathrm{C}$ for $10 \mathrm{~min}$, and blocked with $10 \% \mathrm{BSA}$ in PBS. The fixed cells were then incubated with ANG mAb 26-2F $(5 \mu \mathrm{g} / \mathrm{ml})$. The second antibodies used was Alexa610-conjugated goat anti-mouse $F\left(a b^{\prime}\right)_{2}$ (1:1000 dilution). All images were taken using a Leica TCS SP5 (Wetzlar, Germany) confocal microscope under identical exposure conditions.

qRT-PCR analysis. CDNA was synthesized using Quantitect Reverse Transcription kit from $1 \mu \mathrm{g}$ of DNase-treated total RNA. For ANG and ACTB, oligo dT was used for reverse transcription. For 47S rRNA, the primer for reverse transcription was $5^{\prime}$-CACGAGCCGAGTGATCCAC- $3^{\prime}$. qRT-PCR was carried out using a Light Cycler 480 SYBR Green I Master with the Light Cycler 480 Detection System (Roche Diagnostic, Mannheim, Germany). Cycling conditions were: $95^{\circ} \mathrm{C}$, $5 \mathrm{~min}$; $\left(95^{\circ} \mathrm{C}, 10 \mathrm{~s} ; 60^{\circ} \mathrm{C}, 10 \mathrm{~s}\right)$ repeated for 40 cycles; $72{ }^{\circ} \mathrm{C}, 15 \mathrm{~s}$. ANG primers used for the PCR were designed using the Primer Designer 2.0 software (CLC bio, Cambridge, MA, USA) with the following sequences: forward, $5^{\prime}$-AGAAGCGGGT GAGAAACAA- $3^{\prime}$; and reverse, $5^{\prime}$-TGTGGCTCGGTACTGGCATG- $3^{\prime}$. The primers for 47S rRNA were: forward, 5'-CTCGCCAAATCGACCTCGTA-3'; and reverse, $5^{\prime}$-CACGAGCCGAGTGATCCAC- $3^{\prime} .{ }^{48}$ Actin primer sequences were: forward, $5^{\prime}$-A TCACTATTGGCAACGAGC-3'; and reverse, 5'-GGTCTTTACGGATGTCAACG-3'. qRT-PCR experiments were performed in triplicate, and the results were analyzed using the comparative $\mathrm{Ct}$ method normalized against the housekeeping gene ACTIN. The range of ANG expression levels was determined by calculating the S.D. of the $\Delta \mathrm{Ct}$.

EB/AO staining of apoptotic cells. Cells were detached by trypsinization, centrifuged, and washed in ice-cold PBS. Cells were re-suspended in $50 \mu \mathrm{l}$ of PBS containing the EB-AO dye mixture $(5 \mu \mathrm{g} / \mathrm{ml})$ for $15 \mathrm{~min}$ at $37^{\circ} \mathrm{C}$. Stained cells were placed on a clean microscope slide and covered with coverslips. A total of 750 cells were counted for each group.

\section{Conflict of Interest}

The authors declare no conflict of interest

Acknowledgements. This work was supported in part by the Italian Ministry of University and by the NIH grants R01 NS065237 and R01 CA105241 (to G-FH). Confocal microscopy investigations were carried out at the CISME (Interdepartmental Centre of Electronic Microscopy) of the University of Naples Federico II.

1. Fielding CJ, Fielding PE. Molecular physiology of reverse cholesterol transport. J Lipid Res 1995; 36: 211-228.

2. Pastore L, Belalcazar LM, Oka K, Cela R, Lee B, Chan L et al. Helper-dependent adenoviral vector-mediated long-term expression of human apolipoprotein A-I reduces atherosclerosis in apo E-deficient mice. Gene 2004; 327: 153-160.

3. Brouillette CG, Anantharamaiah GM, Engler JA, Borhani DW. Structural models of human apolipoprotein A-l: a critical analysis and review. Biochim Biophys Acta 2001; 1531: 4-46. 
4. Obici L, Franceschini G, Calabresi L, Giorgetti S, Stoppini M, Merlini G et al. Structure function and amyloidogenic propensity of apolipoprotein A-I. Amyloid 2006; 13: 191-205.

5. Rowczenio D, Dogan A, Theis JD, Vrana JA, Lachmann HJ, Wechalekar AD et al Amyloidogenicity and clinical phenotype associated with five novel mutations in apolipoprotein A-I. Am J Pathol 2011; 179: 1978-1987.

6. Bellotti V, Nuvolone M, Giorgetti S, Obici L, Palladini G, Russo P et al. The workings of the amyloid diseases. Ann Med 2007; 39: 200-207.

7. Amarzguioui M, Mucchiano G, Haggqvist B, Westermark P, Kavlie A, Sletten K et al. Extensive intimal apolipoprotein A1-derived amyloid deposits in a patient with an apolipoprotein A1 mutation. Biochem Biophys Res Commun 1998; 242: 534-539.

8. Obici L, Palladini G, Giorgetti S, Bellotti V, Gregorini G, Arbustini E et al. Liver biopsy discloses a new apolipoprotein A-I hereditary amyloidosis in several unrelated Italian families. Gastroenterology 2004; 126: 1416-1422.

9. Li S, Hu GF. Emerging role of angiogenin in stress response and cell survival under adverse conditions. J Cell Physiol 2012; 227: 2822-2826.

10. Moroianu J, Riordan JF. Nuclear translocation of angiogenin in proliferating endothelia cells is essential to its angiogenic activity. Proc Natl Acad Sci USA 1994: 91: 1677-1681.

11. Xu ZP, Tsuji T, Riordan JF, Hu GF. Identification and characterization of an angiogeninbinding DNA sequence that stimulates luciferase reporter gene expression. Biochemistry 2003; 42: 121-128.

12. Kishimoto K, Liu S, Tsuji T, Olson KA, Hu GF. Endogenous angiogenin in endothelial cells is a general requirement for cell proliferation and angiogenesis. Oncogene 2005; 24: 445-456.

13. Pizzo E, Sarcinelli C, Sheng J, Fusco S, Formiggini F, Netti P et al. Ribonuclease/ angiogenin inhibitor 1 regulates stress-induced subcellular localization of angiogenin and controls its growth and survival activities. J Cell Sci 2013; 126: 4308-4319.

14. Yamasaki S, Ivanov P, Hu GF, Anderson P. Angiogenin cleaves tRNA and promotes stress-induced translational repression. J Cell Biol 2009; 185: 35-42.

15. Ivanov $\mathrm{P}$, Emara MM, Villen J, Gygi SP, Anderson P. Angiogenin-induced tRNA fragments inhibit translation initiation. Mol Cell 2011; 43: 613-623.

16. Baird SD, Turcotte M, Korneluk RG, Holcik M. Searching for IRES. RNA 2006; 12: 1755-1785.

17. Li S, Hu GF. Angiogenin-mediated rRNA transcription in cancer and neurodegeneration. Int J Biochem Mol Biol 2010; 1: 26-35.

18. Greenway MJ, Andersen PM, Russ C, Ennis S, Cashman S, Donaghy C et al. ANG mutations segregate with familial and 'sporadic' amyotrophic lateral sclerosis. Nat Genet 2006; 38: 411-413.

19. Wu D, Yu W, Kishikawa H, Folkerth RD, lafrate AJ, Shen Y et al. Angiogenin loss-offunction mutations in amyotrophic lateral sclerosis. Ann Neurol 2007; 62: 609-617.

20. van Es MA, Schelhaas HJ, van Vught PW, Ticozzi N, Andersen PM, Groen EJ et al. Angiogenin variants in Parkinson disease and amyotrophic lateral sclerosis. Ann Neurol 2011; 70: 964-973

21. Kim YN, Kim DH. Decreased serum angiogenin level in Alzheimer's disease. Prog Neuropsychopharmacol Biol Psychiatry 2012; 38: 116-120.

22. Bai J, Cederbaum Al. Cycloheximide protects HepG2 cells from serum withdrawal-induced apoptosis by decreasing p53 and phosphorylated p53 levels. J Pharmacol Exp Ther 2006 319: $1435-1443$.

23. Ribble D, Goldstein NB, Norris DA, Shellman YG. A simple technique for quantifying apoptosis in 96-well plates. BMC Biotechnol 2005; 5: 12

24. Tsuji T, Sun Y, Kishimoto K, Olson KA, Liu S, Hirukawa S et al. Angiogenin is translocated to the nucleus of HeLa cells and is involved in ribosomal RNA transcription and cell proliferation. Cancer Res 2005; 65: 1352-1360.

25. Yoshioka N, Wang L, Kishimoto K, Tsuji T, Hu GF. A therapeutic target for prostate cancer based on angiogenin-stimulated angiogenesis and cancer cell proliferation. Proc Natl Acad Sci USA 2006; 103: 14519-14524.

26. Moroianu J, Riordan JF. Identification of the nucleolar targeting signal of human angiogenin. Biochem Biophys Res Commun 1994; 203: 1765-1772.

27. Shapiro R, Vallee BL. Site-directed mutagenesis of histidine-13 and histidine-114 of human angiogenin. Alanine derivatives inhibit angiogenin-induced angiogenesis. Biochemistry 1989; 28: 7401-7408.

28. Hallahan TW, Shapiro R, Vallee BL. Dual site model for the organogenic activity of angiogenin. Proc Natl Acad Sci USA 1991; 88: 2222-2226.
29. Li S, Hu MG, Sun Y, Yoshioka N, Ibaragi S, Sheng J et al. Angiogenin mediates androgen-stimulated prostate cancer growth and enables castration resistance. Mol Cancer Res 2013; 11: 1203-1214.

30. Sheng J, Yu W, Gao X, Xu Z, Hu GF. Angiogenin stimulates ribosomal RNA transcription by epigenetic activation of the ribosomal DNA promoter. J Cell Physiol 2014; 229: 521-529.

31. Tesh VL. The induction of apoptosis by Shiga toxins and ricin. Curr Top Microbiol Immunol 2012; 357: 137-178.

32. Marchesi M, Parolini C, Valetti C, Mangione P, Obici L, Giorgetti S et al. The intracellular quality control system down-regulates the secretion of amyloidogenic apolipoprotein A-I variants: a possible impact on the natural history of the disease. Biochim Biophys Acta 2011; 1812: 87-93

33. Comai L. The nucleolus: a paradigm for cell proliferation and aging. Braz J Med Biol Res 1999; 32: 1473-1478.

34. Stoykova AS, Dabeva MD, Dimova RN, Hadjiolov AA. Ribosome biogenesis and nucleolar ultrastructure in neuronal and oligodendroglial rat brain cells. J Neurochem 1985; 45 1667-1676.

35. Graber TE, Lewis SM, Holcik M. An approach to whole-genome identification of IRES elements. Curr Genomics 2006; 7: 205-215.

36. Emara MM, Ivanov P, Hickman T, Dawra N, Tisdale S, Kedersha N et al. Angiogenininduced tRNA-derived stress-induced RNAs promote stress-induced stress granule assembly. J Biol Chem 2010; 285: 10959-10968.

37. Kim HM, Kang DK, Kim HY, Kang SS, Chang SI. Angiogenin-induced protein kinase B/Akt activation is necessary for angiogenesis but is independent of nuclear translocation of angiogenin in HUVE cells. Biochem Biophys Res Commun 2007; 352: 509-513.

38. Trouillon R, Kang DK, Park H, Chang SI, O'Hare D. Angiogenin induces nitric oxide synthesis in endothelial cells through PI-3 and Akt kinases. Biochemistry 2010; 49: 3282-3288.

39. Kieran D, Sebastia J, Greenway MJ, King MA, Connaughton D, Concannon CG et al. Control of motoneuron survival by angiogenin. J Neurosci 2008; 28: 14056-14061.

40. Yacoubian TA, Cantuti-Castelvetri I, Bouzou B, Asteris G, McLean PJ, Hyman BT et al. Transcriptional dysregulation in a transgenic model of Parkinson disease. Neurobiol Dis 2008; 29: 515-528.

41. Kontopoulos E, Parvin JD, Feany MB. Alpha-synuclein acts in the nucleus to inhibit histone acetylation and promote neurotoxicity. Hum Mol Genet 2006; 15: 3012-3023.

42. Li F, Macfarlan T, Pittman RN, Chakravarti D. Ataxin-3 is a histone-binding protein with two independent transcriptional corepressor activities. J Biol Chem 2002; 277 45004-45012

43. McLaughlin RL, Phukan J, McCormack W, Lynch DS, Greenway M, Cronin S et al. Angiogenin levels and ANG genotypes: dysregulation in amyotrophic lateral sclerosis. PLoS One 2010; 5: e15402.

44. Shapiro R, Fox EA, Riordan JF. Role of lysines in human angiogenin: chemical modification and site-directed mutagenesis. Biochemistry 1989; 28: 1726-1732.

45. Shapiro R, Harper JW, Fox EA, Jansen HW, Hein F, Uhlmann E. Expression of Met-(-1) angiogenin in Escherichia coli: conversion to the authentic less than Glu-1 protein. Anal Biochem 1988; 175: 450-461.

46. Shapiro $\mathrm{R}$, Vallee BL. Identification of functional arginines in human angiogenin by site-directed mutagenesis. Biochemistry 1992; 31: 12477-12485.

47. Busch H, Muramatsu M, Adams H, Steele WJ, Liau MC, Smetana K. Isolation of nucleoli. Exp Cell Res 1963; 24(suppl9): 150-163.

48. Monti DM, Yu W, Pizzo E, Shima K, Hu MG, Di Malta C et al. Characterization of the angiogenic activity of zebrafish ribonucleases. FEBS J 2009; 276: 4077-4090.

(c) (1) $\circledast \circledast$ Cell Death and Disease is an open-access journal published by Nature Publishing Group. This work is licensed under a Creative Commons Attribution-NonCommercialNoDerivs 3.0 Unported License. To view a copy of this license, visit http://creativecommons.org/licenses/by-nc-nd/3.0/

\section{Supplementary Information accompanies this paper on Cell Death and Disease website (http://www.nature.com/cddis)}

